\title{
Europe unveils risk assessment guidance for engineered nanomaterials used in food chain
}

$\mathrm{W}$ ith food additives, flavourings, enzymes and other socalled "engineered nanomaterials" becoming altogether more common in products on market shelves, the Parma, Italy-based European Food Safety Authority has issued the world's first guidance on determining the potential health risks they pose.

The "Guidance on the risk assessment of the application of nanoscience and nanotechnologies in the food and feed chain," prepared at the request of the European Commission, sketches the parameters for scientific risk assessments to be made of engineered nanomaterials, including their identification, chemical characterization and toxicity (www.efsa.europa.eu/en/efsa journal/doc/2140.pdf).

The guidance includes recommendations regarding "the physico-chemical characterisation requirements of engineered nanomaterials used e.g. as food additives, enzymes, flavourings, food contact materials, novel foods, feed additives and pesticides and; testing approaches to identify and characterise hazards arising from the nanoproperties which, in general, should include information from in vitro genotoxicity, absorption, distribution, metabolism and excretion and repeated dose 90-day oral toxicity studies in rodents."

The guidance essentially proposes different levels of toxicity testing and reporting depending on the level of exposure to engineered nanomaterials. In cases where people will digest nanomaterials, it recommends that toxicity tests be done through both in-vitro and in-vivo experiments to determine the estimated safe level of human consumption. Experimental data show that engineered nanomaterials seem to accumulate mainly in the mononuclear phagocyte system tissues, particularly the spleen, liver and the gastrointestinal tract.

risks that nanotechnology applications may bring to the food sector. There are still no state-of-the-art or foreseeable applications to highlight opportunities for innovation. We all need to know the areas of uncertainty in relation to public perception of the new technological developments and potential implications for consumer safety and current regulatory controls."

But the need for guidance in the area is self-evident given the expected increase in the use of engineered nanomaterials in the future, Dr. David Carlander, scientific officer of the European Food Safety Authority, writes in an email. "A rise of applications concerning nanomaterials in different food and feed sectors is expected both inside and outside the European Union. This is due to

The report acknowledges that uncertainties remain at all levels of the risk assessment process, including the identification, characterization and detection of engineering nanomaterials, because test methods are not yet finetuned. Moreover, the applicability of many of the current biological and toxicological testing methods that suit nonnanoform substances may not be suitable for engineered nanomaterials.

Such uncertainties surround applications of nanoscience and nanotechnology in food, Paula Ravasco, lead researcher in the metabolism and nutrition unit at the Institute of Molecular Medicine at the University of Lisbon, Portugal, writes in an email. "There is a great gap in information, and up-to-date information is much needed on the products and applications of nanotechnology for the food sector - for scientists, regulators and consumers alike. We all need an independent, balanced and impartial view of the potential benefits as well as the considerable amount of applied research being performed in academia and industry. The advantages of these applications will depend on the type of application. Nanotechnology applications in foods may, for example, modify taste, texture, consistency, fat content, nutrient absorption and bioavailability. For instance, a food may have a reduced fat content while retaining the mouth-feel of a fatty food. Or an easily degradable nutrient can be protected during the passage through the acidic environment of the stomach."

Ravasco says that research is now being "conducted to develop nanocapsules containing nutrients that would be released when nanosensors detect a vitamin deficiency in the body. And 'interactive' foods are being developed that would allow the person to choose the desired flavour and colour." - Tiago Villanueva, MD, Lisbon, Portugal 